\title{
Óbitos por serpentes peçonhentas no Estado de São Paulo: avaliação de 43 casos, 1988/93
}

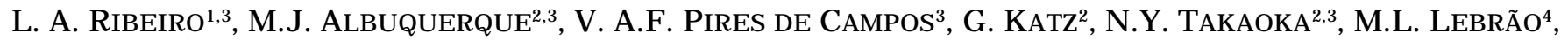 \\ M.T. J ORGE ${ }^{1}$
}

Departamento de Clínica Médica da Universidade Federal de Uberlândia, Uberlândia, MG ${ }^{1}$; Centro de Vigilância Epidemiológica AlexandreVranjac, São Paulo, SP2; Comissão Permanente de Coordenação e Controle dos Aci dentes por Animais Peçonhentos do E stado de São Paulo3; Departamento de E pidemi ologia da Faculdade de Saúde Pública da Universidade de São Paulo, São Paulo, SP4.

RESUMO - São ainda mal estabelecidos os fatores prognósticos para a ocorrência de óbito nos envenenamentos por serpentes e as causas que 0 determinam.

ОвJ eтivo. Determinar, no envenenamento ofídico, os fatores prognósticos e as causas mais prováveis do óbito.

Métodos. No período de 1988 a 1993 foram notificados 12.639 casos de acidentes por serpentes peçonhentas no Estado de São Paulo, com 43 óbitos $(0,34 \%)$. Foram comparadas as variáveis obtidas das fichas de notificação dos acidentes com aquelas dos prontuários e/ou dos relatórios médicos dos casos de óbito.

Resultados. O diagnóstico quanto ao gênero da serpente foi realizado em 11.297 acidentes, sendo $9.828(87 \%)$ por Bothrops, 1.359 (12\%) por Crotalus e 110 (1\%) por Micrurus, e em 41 casos de óbito, sendo $28(68,3 \%)$ por Bothrops e $13(31,7 \%)$ por Crotalus (p<0,05). Informação quanto a sexo e idade dos pacientes foi fornecida em, respectivamente, 12.620 e 12.527 acidentes. Pertenciam ao sexo masculino $9.783(77,5 \%)$ pacientes picados e $35(81,4 \%)$ que faleceram $(p>0,05)$. Tinham 50 anos ou mais $15,9 \%$ dos pacientes picados e $41,8 \%$ daqueles que faleceram $(p<0,05)$. As regiões

\section{NTRODUÇÃO}

No Estado de São Paulo, as serpentes peçonhentas pertencem a três gêneros: Bothrops, Crotalus e Micrurus ${ }^{1}$. Aquelas do gênero Bothrops são as principais responsáveis por acidentes, e as do gênero Micrurus raramente os causam².

O envenenamento por Bothrops pode levar a intensa inflamação na região da picada, com grande destruição tecidual, o que, muitas vezes, se complica com infecção por bactérias provenientes do próprio veneno da serpente ${ }^{3,4}$. E nvenenamentos por Bothrops e por Crotalus levam a alteração da coagulação sanguínea, o que facilita a ocorrência de sangramento. No envenenamento por Crotalus anatômicas mais freqüentemente picadas foram: pé $(42,2 \%)$, mão $(20,6 \%)$, perna $(17,6 \%)$ e tornozelo $(13,1 \%)$, nos acidentes, e pé $(35,7 \%)$ e perna $(35,7 \%)$, nos casos de óbito $(p<0,05)$. A alteração da coagulação ocorreu em 34 (91,9\%) de 37 pacientes que evoluíram para o óbito. As manifestações e complicações mais referidas como possíveis causas de óbito foram: insuficiência renal $(34-79,1 \%)$, insuficiência respiratória (28- 65,1\%), choque (1841,9\%) e septicemia (18- 41,9\%). Dentre os pacientes que evoluíram para óbito, excluindo-se um caso sem informação, $29,4 \%$ faleceram dentro dos primeiros dois dias após a picada. A insuficiência respiratória foi mais comum entre os que faleceram devido ao envenenamento crotálico e a septicemia só foi referida para o envenenamento botrópico.

Conclusão. A maioria dos acidentes e dos óbitos é causada por Bothrops; são mais freqüentemente letais os envenenamentos por Crotalus, as picadas na perna e em pessoas com mais de 50 anos; a complicação mais comum nos casos fatais é a insuficiência renal.

UNITERMOS: Acidente ofídico. Quadro clínico. Óbito.

e por Micrurus, pode ocorrer paralisia flácida da musculatura esquelética, principalmente a ocular extrínseca, facial e, às vezes, da respiração, com conseqüente insufici ência respiratória. No acidente por Crotalus, ocorre também miotoxicidade sistêmica (rabdomiólise, principalmente de fibras do tipo 1), com intensa mi ogl obinúria e insufi ciência renal aguda (IRA) por necrose tubular aguda (NTA). No envenenamento por Bothrops, pode ocorrer IRA por NTA ou por necrose cortical, cuja fisiopatologia é mal conhecida. Pelo menos esses dois últimos envenenamentos podem cursar com choque $^{3,5-7}$. O choque parece poder ser causado pelo próprio veneno, por sangramento ou, no caso de envenenamento botrópico, por septicemia ${ }^{3,8,9}$. 
Desde a década de 40, Barroso, analisando boletins referentes a atendimentos a pacientes picados por serpentes encaminhados ao Instituto Vital Brazil, no Rio de J aneiro, observou correl ação da letalidade com o porte da vítima, sendo as crianças e os adultos do sexo feminino, em ordem decrescente, os mais propensos ao óbito ${ }^{10}$. Entretanto, apesar dos recentes avanços no conhecimento dos envenenamentos por serpentes, ainda não estão bem estabelecidos os fatores prognósticos para a ocorrência de óbito e as causas que mais comumente o determinam.

O presente estudo avalia os acidentes por serpentes peçonhentas e os casos de óbitos por esses envenenamentos ocorridos durante os anos de 1988 a 1993, período propício à obtenção dos dados devido a grande organização do sistema de notificação do acidente ofídico no E stado de São Paulo e da necessidade de se notificarem os casos de envenenamento com uso de soro para se conseguir a reposi ção do estoque ${ }^{2}$. Tem como obj etivo determinar fatores prognósticos para o óbito e suas prováveis causas.

\section{MATERIAL E MÉTODO}

I nformações sobre os acidentes e envenenamentos, ocorridos nos anos de 1988 a 1993, foram obtidas das "Fichas de N otificação de Acidentes Ofídicos no Estado de São Paulo" pertencentes ao sistema de notificação compulsória da Secretaria de Estado da Saúde. Dados sobre os 43 pacientes que evoluíram para óbito foram obtidos da referida ficha e, em 30 deles, também dos prontuários médicos e/ou relatórios médicos, conseguidos por mei o de contatos com autoridades de saúde e com responsáveis pelos Pontos Estratégicos para o Tratamento de Acidentes por Animais Peçonhentos, no Estado de São Paulo.

Foram coletadas as variáveis: sexo e idade dos pacientes, região anatômi ca picada, gênero da serpente, intervalo de tempo entre o acidente e o atendi mento e entre a picada e o óbito, as manifestações graves dos envenenamentos e complicações apresentadas.

As comparações entre as freqüências das variáveis, no grupo de acidentes e no grupo dos casos que evoluíram para óbito, foram feitas pelo teste do $\chi^{2} \mathrm{e}$, quando necessário, pelo teste exato de Fischer.

\section{RESULTADOS}

F oram estudados 12.639 aci dentes notificados e 43 casos de óbito $(0,34 \%)$. O gênero da serpente foi referido em 11.297 acidentes e em 41 dos envene-
Tabela 1 - Acidentes ofídicos segundo evolução e idade dos pacientes, Estado de São Paulo, 1988/93

\begin{tabular}{|c|c|c|c|c|}
\hline \multirow[t]{2}{*}{ Faixa etária } & \multicolumn{2}{|c|}{ Total dos acidentes } & \multicolumn{2}{|c|}{ Óbito } \\
\hline & $n^{\circ}$ & $\%$ & $n^{\circ}$ & $\%$ \\
\hline $0 \vdash 10$ & 1.187 & 9,5 & 5 & 11,6 \\
\hline $10 \vdash 20$ & 2.931 & 23,4 & 5 & 11,6 \\
\hline $20 \vdash 30$ & 2.658 & 21,3 & 6 & 14,0 \\
\hline $30 \vdash 40$ & 2.235 & 17,8 & 3 & 7,0 \\
\hline $40^{\vdash} 50$ & 1.516 & 12,1 & 6 & 14,0 \\
\hline $50 \vdash 60$ & 1.133 & 9,0 & 7 & 16,2 \\
\hline$\geqq \vdash 60$ & 867 & 6,9 & 11 & 25,6 \\
\hline Total & $12.527^{*}$ & 100,0 & 43 & 100,0 \\
\hline
\end{tabular}

Tabela 2 - Acidentes ofídicos segundo evolução e região anatômica picada, Estado de São Paulo, 1988/93

\begin{tabular}{|c|c|c|c|c|}
\hline \multirow[t]{2}{*}{ Região anatômica } & \multicolumn{2}{|c|}{ Total de acidentes* } & \multicolumn{2}{|c|}{ Óbito } \\
\hline & $\mathrm{n}^{\circ}$ & $\%$ & $\mathrm{n}^{\circ}$ & $\%$ \\
\hline Membros inferiores & 9.456 & 75,3 & 34 & 81,0 \\
\hline pé & 5.299 & 42,2 & 15 & 35,7 \\
\hline tornozelo & 1.647 & 13,1 & 3 & 4,1 \\
\hline perna & 2.214 & 17,6 & 15 & 35,7 \\
\hline joelho/coxa & 186 & 1,5 & 1 & 2,4 \\
\hline não especificado & 110 & 0,9 & 0 & 0,0 \\
\hline Membros superiores & 2.981 & 23,7 & 7 & 16,6 \\
\hline mão & 2.584 & 20,6 & 3 & 7,1 \\
\hline punho/antebraço & 226 & 1,8 & 3 & 7,1 \\
\hline cotovelo/braço & 153 & 1,2 & 0 & 0,0 \\
\hline não especificado & 18 & 0,1 & 1 & 2,4 \\
\hline Cabeça/pescoço & 56 & 0,4 & 0 & 0,0 \\
\hline Tronco & 60 & 0,5 & 1 & 2,4 \\
\hline Total & $12.553^{*}$ & 100,0 & $42^{*}$ & 100,0 \\
\hline
\end{tabular}

namentos fatais. Em ordem decrescente de freqüência, Bothrops foi responsável por 9.828 (87,0\%) acidentes e 28 (68,3\%) óbitos, Crotalus por 1.359 (12\%) acidentes e 13 óbitos (31,7\%) e Micrurus por 110 (1\%) acidentes e nenhum óbito $(p<0,05)$. A informação quanto a sexo e idade dos pacientes foi fornecida em, respectivamente, 12.620 e 12.527 acidentes e em todos aqueles de evolução fatal. Pertenciam ao sexo masculino $9.783(77,5 \%)$ pacientes entre os casos de acidentes e $35(81,4 \%)$ entre os casos de óbito $(p>0,05)$. A distribui ção do número de acidentes e de óbitos de acordo com a faixa etária do paciente é mostrada na tabela 1 , onde pode ser observado que apenas $15,9 \%$ dos pacientes picados mas $41,8 \%$ daqueles que faleceram tinham 50 anos ou mais $(p<0,05)$. 
As regi ões anatômicas mais freqüentemente picadas foram: pé $(42,2 \%)$, mão $(20,6 \%)$, perna $(17,6 \%)$ e tornozel o $(13,1 \%)$, nos casos de acidentes, e pé $(35,7 \%)$ e perna ( $35,7 \%)$, nos casos de óbito (tabela 2). Observou-se associação entre pi cada na perna e ocorrência de óbito $(p<0,05)$.

O tempo entre o aci dente e a assistência médica no serviço de saúde que o notificou foi obtido em 12.366 casos e em 41 envenenamentos fatais. Foram atendidos dentro de três e seis horas após a picada, respectivamente, $73,2 \%$ e $89 \%$ dos pacientes picados e $41,4 \%$ e $63,4 \%$ dos que faleceram $(p<0,05)$.

Houve avaliação da coagulação sanguínea em 37 pacientes que faleceram e em 34 (91,9\%) ela estava alterada. Essa informação não foi obtida nos casos de acidente não-fatal. As manifestações e complicações mais referidas como possíveis causas de óbito foram, isolada ou conjuntamente, a insuficiência renal (79,1\%), insuficiência respiratória (65,1\%), septicemia (41,9\%) e choque $(41,9 \%)$. Os pacientes que apresentaram choque faleceram entre 10 horas e 24 dias (mediana de aproximadamente três dias) após a picada.

Nos casos de óbito não houve diferença marcante na freqüência de picadas nas diferentes regiões anatômicas, entre os acidentes por Bothrops e aqueles por Crotalus (tabela 3 ).

Os 28 pacientes que faleceram por envenenamento botrópico apresentaram: bolha (8-28,6\%), necrose (17-60,7\%), abscesso (12-42,9\%), síndrome compartimental (6-21,4\%), trombose venosa profunda (4-14,3\%), vômito (7-25\%), sangramento (14-50\%), choque (15-53,6\%), insuficiência renal (23-82,1\%), insuficiência respiratória (15-53,6\%), pneumonia (2-7,1\%), embolia pulmonar (3-10,7\%), septicemia (7-25,0\%), coagulação intravascular disseminada (6-21,4\%), úlcera de estresse (1-3,6\%), confusão mental (8-28,6\%), crise convulsiva (2$7,1 \%$ ), acidente vascular cerebral hemorrágico (4$14,3 \%$ ), coma (4-14,3\%); em três casos o choque foi referido como tendo si do causado por septicemia e em um por hemorragia; em três casos de insuficiência respiratória foi referida ocorrência de edema agudo de pulmão; a alteração da coagulação sanguínea ocorreu em todos os 23 (100\%) casos em que esse dado foi referido. Foram realizados os seguintes procedimentos: diálise (6-21,4\%), fasciotomia (5-17,9\%) e traqueostomia (1-3,6\%).

Os 13 pacientes que faleceram por envenenamento crotálico apresentaram: vômito (3 - 23,1\%), mialgia (6-46,2\%), mioglobinúria (11-84,6\%), sangramento $(3-23,1 \%)$ choque $(7-53,8 \%)$, insuficiência renal (11-84,6\%), anasarca (2-15,4\%), insuficiência respiratória (13-100\%), coma (6-46,2\%),

\begin{tabular}{|c|c|c|c|c|c|c|c|c|}
\hline \multirow{3}{*}{$\begin{array}{c}\text { Região } \\
\text { anatômica } \\
\text { picada }\end{array}$} & \multicolumn{6}{|c|}{ Gênero da serpente } & & \\
\hline & \multicolumn{2}{|c|}{ Botrópico } & \multicolumn{2}{|c|}{ Crotálico } & \multicolumn{2}{|c|}{ NR } & \multicolumn{2}{|c|}{ Total } \\
\hline & no & $\%$ & no & $\%$ & & $\%$ & no & $\%$ \\
\hline Membros inf. & 21 & 75,0 & 11 & 91,7 & & 100,0 & 34 & 81,0 \\
\hline pé/tornozelo & 11 & 39,3 & 6 & 50,0 & 1 & 50,0 & 18 & 42,9 \\
\hline perna & 9 & 32,1 & 5 & 41,7 & 1 & 50,0 & 15 & 35,7 \\
\hline coxa & 1 & 3,6 & - & - & - & - & 1 & 2,4 \\
\hline Membros sup. & 6 & 21,4 & 1 & 8,3 & - & - & 7 & 16,6 \\
\hline mão & 3 & 10,7 & - & - & - & - & 3 & 7,1 \\
\hline antebraço & 2 & 7,1 & 1 & 8,3 & - & - & 3 & 7,1 \\
\hline não referido & 1 & 3,6 & - & - & - & - & 1 & 2,4 \\
\hline Tronco & 1 & 3,6 & - & - & - & - & 1 & 2,4 \\
\hline Total & 28 & 100,0 & 12 & 100,0 & 2 & 100,0 & $42^{*}$ & 100,0 \\
\hline
\end{tabular}

$\mathrm{NR}=$ não referido.

* Em um acidente crotálico não havia informação quanto à região anatômica picada.

Tabela 4 - Distribuição dos óbitos segundo o gênero da serpente e a faixa etária dos pacientes, Estado de São Paulo, 1988/93

\begin{tabular}{|c|c|c|c|c|c|c|c|c|}
\hline \multirow{3}{*}{$\begin{array}{c}\text { Faixa } \\
\text { etária do } \\
\text { paciente }\end{array}$} & \multicolumn{6}{|c|}{ Gênero da serpente } & & \\
\hline & \multicolumn{2}{|c|}{ Botrópico } & \multicolumn{2}{|c|}{ Crotálico } & \multicolumn{2}{|c|}{ NR } & \multicolumn{2}{|c|}{ Total } \\
\hline & no & $\%$ & no & $\%$ & no & $\%$ & no & $\%$ \\
\hline $0 \vdash 10$ & 2 & 7,1 & 3 & 23,1 & - & - & 5 & 11,6 \\
\hline $10 \vdash 20$ & 3 & 10,7 & 2 & 15,4 & - & - & 5 & 11,6 \\
\hline $20 \vdash 30$ & 6 & 21,5 & - & - & - & - & 6 & 14,0 \\
\hline $30 \vdash 40$ & 2 & 7,1 & 1 & 7,7 & - & - & 3 & 7,0 \\
\hline $40 \vdash 50$ & 1 & 3,6 & 4 & 30,7 & 1 & 50,0 & 6 & 14,0 \\
\hline $50 \vdash 60$ & 4 & 14,3 & 2 & 15,4 & 1 & 50,0 & 7 & 16,2 \\
\hline$\geqq 60$ & 10 & 35,7 & 1 & 7,7 & - & - & 11 & 25,6 \\
\hline Total & 28 & 100,0 & 13 & 100,0 & 2 & 100,0 & 43 & 100,0 \\
\hline
\end{tabular}

pneumonia (3-23,1\%); em três casos de insuficiência respiratória essa foi referida como tendo sido causada por edema pulmonar; a alteração da coagulação sanguínea ocorreu em dez $(83,3 \%)$ de 12 casos em que o dado foi obtido. Foram submetidos aos seguintes procedimentos: diálise $(3-23,1 \%)$, transfusão sanguínea (2-15,4\%), sangria $(2-15,4 \%)$ e traqueostomia (1-7,7\%). A maior freqüência de óbitos entre pacientes com 50 anos ou mais, em comparação com a freqüência de casos não-fatais, nessa faixa etária, não pôde ser observada no envenenamento crotálico (tabelas 1 e 4).

Os paci entes que faleceram devido a envenenamento botrópico receberam 20 ou mais ampolas de soro em $11,1 \%$ dos casos e aqueles que faleceram por envenenamento crotálico receberam essa dose em 53,8\% ( $p<0,05)$ (tabela 5). Evoluíram para óbito, dentro de 48 horas após a picada, 29,4\% dos pacientes e, nos primeiros cinco dias, $67,6 \%$. Não 
Tabela 5 - Distribuição dos óbitos segundo o gênero da serpente e a dose de soro administrada, Estado de São Paulo, 1988/93

\begin{tabular}{|c|c|c|c|c|c|}
\hline \multirow{3}{*}{$\begin{array}{c}\text { Dose } \\
\text { (número } \\
\text { de ampolas) }\end{array}$} & \multicolumn{4}{|c|}{ Acidente } & \multirow[b]{2}{*}{ Total } \\
\hline & \multicolumn{2}{|c|}{ Botrópico } & Crotálico & NR & \\
\hline & $\mathrm{n}^{\circ}$ & $\%$ & $\begin{array}{ll}\mathbf{n}^{\circ} & \%\end{array}$ & $\mathrm{n}^{\mathrm{o}}$ & $\overline{\text { no }} \quad \%$ \\
\hline$<5$ & 1 & 3,7 & 7,7 & - & 4,9 \\
\hline $5 \vdash 10$ & 4 & 14,8 & - & - & $4 \quad 9,8$ \\
\hline $10 \vdash 20$ & 19 & 70,4 & 538,5 & - & 2458,5 \\
\hline$\geqq 20$ & 3 & 11,1 & $7 \quad 53,8$ & 1100,0 & 1126,8 \\
\hline Total & $27^{*}$ & 100,0 & 13100,0 & $1^{* *} 100,0$ & 41100,0 \\
\hline \multicolumn{6}{|c|}{$\begin{array}{l}\text { * Em um caso de óbito por envenenamento botrópico o paciente não } \\
\text { recebeu soro por não ter sido feito diagnóstico precoce. }\end{array}$} \\
\hline \multicolumn{6}{|c|}{$\begin{array}{l}{ }^{* *} \text { Um dos pacientes que faleceu por picada de serpente não identificada } \\
\text { não chegou a receber soro, pois não chegou vivo ao hospital. }\end{array}$} \\
\hline
\end{tabular}

Tabela 6-Óbito por acidentes ofídicos segundo o intervalo de tempo em que ocorreu, após o acidente, Estado de São Paulo, $1988 / 93$

\begin{tabular}{|c|c|c|c|c|c|c|c|c|}
\hline \multirow{3}{*}{$\begin{array}{c}\text { Intervalo } \\
\text { (dias) }\end{array}$} & \multicolumn{6}{|c|}{ Acidente } & & \\
\hline & \multicolumn{2}{|c|}{ Botrópico } & \multicolumn{2}{|c|}{ Crotálico } & \multicolumn{2}{|c|}{ NR } & \multicolumn{2}{|c|}{ Total } \\
\hline & $\mathrm{n}^{\mathrm{o}}$ & $\%$ & $\overline{n^{\circ}}$ & $\%$ & no & $\%$ & $\overline{\text { no }^{\circ}}$ & $\%$ \\
\hline$<1$ & 2 & 9,5 & 1 & 8,4 & 1 & 100,0 & 4 & 11,8 \\
\hline $1 \vdash 2$ & 3 & 14,3 & 3 & 25,0 & - & - & 6 & 17,6 \\
\hline $2 \vdash 5$ & 9 & 42,9 & 4 & 33,3 & - & - & 13 & 38,2 \\
\hline$\geqq 5$ & 7 & 33,3 & 4 & 33,3 & - & - & 11 & 32,4 \\
\hline Total & $21^{*}$ & 100,0 & 12 & 100,0 & 1 & 100,0 & $34^{*}$ & 100,0 \\
\hline
\end{tabular}

houve diferença nesse tempo de evolução entre os pacientes picados por Bothrops e por Crotalus (tabela 6).

\section{DISCUSSÃo}

As serpentes do gênero Bothrops foram as principais causadoras de acidentes e de óbitos, mas a porcentagem de picada de Crotalus que evoluiu para óbi to foi maior, o que reforça o conhecimento da mai or letalidade do acidente crotálico em relação ao botrópico ${ }^{2,6,11}$. O acidente por Micrurus foi raro no Estado de São Paulo, o que corrobora achados anteriores ${ }^{2,9}$. Embora esse envenenamento seja considerado sempre grave pelos manuais oficiais de atendimento de acidentes por animais peçonhentos ${ }^{5,7}$, pequena série de casos já havia colocado em dúvida essa informação $0^{3,12}$. No presente estudo, dos 110 pacientes picados por Micrurus nenhum faleceu. Mesmo que alguns desses casos representem erros diagnósticos, a ausência de óbito mostra que o envenenamento por coral não é tão grave quanto havia sido sugerido ${ }^{5}$.
No presente estudo não foi demonstrada, ao contrário de estudo anterior ${ }^{10,13}$, mai or gravidade do acidente com crianças e com pessoas do sexo feminino. Obteve-se, no entanto, associação da evolução fatal com o envenenamento em pessoas mais idosas (50 anos e mais). I sso, provavel mente, se deve ao estado de saúde dessas pessoas que podem apresentar, previamente, doenças que acometem órgãos, tais como rins e pulmões, que são comumente lesados pelo veneno.

Picadas na perna foram mais comuns entre os casos fatais, sugerindo que essa variável esteja relacionada à gravidade do envenenamento. Avaliações apenas da gravidade quanto à ocorrência de necrose, abscesso e amputação já mostraram associação positiva com a picada na perna ${ }^{14}$. Isso talvez se deva a grande extensão de superfície e quantidade de tecido dessa região, o que facilita a introdução dos dois dentes inoculadores da serpente e a maior inoculação de veneno mas, também, pelo menos em parte, a maior freqüência de picadas na perna por serpentes de grande porte ${ }^{11}$.

Os pacientes que faleceram foram atendi dos, em média, mais tardiamente do que os demais. I sso sugere que o tratamento tardio seja fator de mau prognóstico, o que é plenamente explicável, pois o soro neutraliza o veneno e, portanto, deve ser administrado antes que ele tenha desenvolvido a sua ação. Cuidados como a hidratação também podem ser mais útei s se ini ci ados precocemente ${ }^{3,15}$. Entretanto, pacientes com envenenamento grave, devido à evol ução desfavorável, quando não procuram atendimento precocemente, podem vir a fazê-lo posteriormente, o que dificilmente ocorreria com aqueles com envenenamentos de pequena gravidade. Além disso, os casos mais graves são muitas vezes transferidos da unidade de atendimento inicial para outras de maior complexidade e, às vezes, são notificados como tendo sido atendidos tardiamente pelos serviços para os quais foram encaminhados.

A alteração da coagulação sanguínea, presente em cerca de $90 \%$ dos pacientes que evoluíram para óbito, sugere sua relação com a gravidade do envenenamento por serpente peçonhenta. Tem sido demonstrado que essa percentagem é menor, tanto nos casos de acidente botrópico em geral ${ }^{16}$ quanto nos casos de acidentes por $B$. jararaca ${ }^{11}$ ou por Crotalus durissus ${ }^{6}$.

A pesar de ter sido avaliado o prontuário médico e/ou relatório médico de 30 dos 43 casos de óbito, na maioria das vezes sua causa não foi determinada com exatidão. Isto deveu-se à precariedade das informações neles contidas, o que é esperado em países do ter ceiro mundo ${ }^{17}$, mas, também, à compl exidade 
fisiopatológica dos envenenamentos por serpentes peçonhentas ${ }^{3,7}$. Pode-se observar, apesar disso, que a mani festação mais comumente relatada, que poderia ter sido a causa do óbito, foi a IRA, o que é concordante com os dados da literatura ${ }^{3,18}$. A IRA e o choque foram igualmente freqüentes entre pacientes picados por Bothrops e por Crotalus. Já tem sido referido na literatura que a IRA é a principal causa de óbito, tanto no acidente botrópico como no crotálico ${ }^{3,18}$, e que o choque pode ocorrer nesses dois envenenamentos ${ }^{9}$. O longo tempo transcorrido entre o acidente e o óbito, nos pacientes que apresentaram choque, sugere que ele não foi a causa imediata da morte ou que não foi diretamente causado pelo veneno. A insuficiência respiratória foi, também, freqüente em ambos os envenenamentos, mas ocorreu em pouco mais da metade dos casos de picadas por Bothrops e em todos aqueles por Crotalus. Nesses dois grupos houve descrição de edema agudo de pulmão, cuja fisiopatologia não se conhece. A maior freqüência dessa insuficiência no envenenamento crotálico é esperada, uma vez que o veneno causa rabdomiólise generalizada ${ }^{3,19}$ e, principalmente, paralisia flácida, com conseqüente insuficiência respiratória restritiva ${ }^{6,18}$. A septicemia foi referida em um quarto dos acidentes por Bothrops e não foi referida naqueles por Crotalus e a pneumonia em cerca de um quarto daquel es picados por Crotalus e em menos de $10 \%$ daqueles por Bothrops. A ocorrência de septicemia no envenenamento botrópico, em parte, pode ser explicada pela disseminação das bactérias que causam infecção na região da picada e a maior freqüência de pneumonia no envenenamento crotálico, talvez, pela maior proporção de pacientes que necessitam assistência ventilatória e que poderiam desenvolver pneumonia nosocomial. O intervalo de tempo entre a picada e o óbito foi maior do que dois dias, na maioria dos casos, e maior do que cinco dias, em quase um terço deles. Isso sugere ter havi do tempo suficiente para ocorrerem complicações, como infecções hospitalares, principal mente entre pacientes com insuficiência respiratória, que acabam por ser submetidos a procedi mentos de risco ${ }^{20}$. Esse período é também suficiente para uma boa avaliação dos pacientes e estabelecimento de terapêutica adequada, o que indica, também, que uma assistência médica eficiente pode vir a modificar o curso clínico dos envenenamentos graves.

Relatos de complicações, como coagulação intravascular disseminada (CIVD) e trombose venosa profunda (TVP), devem ser interpretados com cuidado, pois o veneno costuma causar coagul opatia de consumo e pode determinar intenso edema inflamatório³.
Tais manifestações podem levar a falso diagnóstico de CIVD e TVP, respectivamente. É também difícil a avaliação das causas da insuficiência respiratória que, na maioria dos casos do presente estudo, não teve sua etiologia definida. Choque, septicemia, insuficiência respiratória e, principalmente, IRA têm sido referidos como causas de óbi to no envenenamento ofídico 3,18,21. O veneno da maioria das serpentes possui muitas toxinas e, portanto, atua em muitos órgãos e causa lesões por múltiplos mecanismos ${ }^{3}$, o que explica a diversidade de manifestações graves encontradas. Fazem exceção as serpentes do gênero Micrurus, que, como manifestação importante, costumam causar apenas paralisia flácida dos músculos esqueléticos ${ }^{22}$.

Entre os pacientes que fal eceram por envenenamento botrópico, a ocorrência de bolha, necrose e abscesso foi maior do que a anteriormente verificada para o acidente por Bothrops no Estado de São Paulo, conforme atendimento do Hospital Vital Brazil ${ }^{16}$, e, especificamente, naqueles por $B$. jarara$c a^{11}$ ou por $B$. moojeni ${ }^{23}$, espécies de serpentes do gênero Bothrops comumente causadoras de acidentes nesse Estado. I sso sugere associação entre a intensidade das manifestações locais e a evolução para o óbito. Estudo realizado com base no atendimento do Hospital Vital Brazil do Instituto Butantan também mostrou associação entre tais manifestações locais (bol ha, necrose e abscesso) e o óbito ${ }^{24}$.

A alteração na coagulação sanguínea foi mais freqüente nos casos de óbito por Bothrops (100\% dos casos para os quais havia a informação) do que o que é conhecido para o envenenamento por serpentes, desse gênero, que costumam ocorrer em São Paulo ${ }^{3,24}$. Entretanto, não tem sido observada associação entre alteração da coagulação sanguínea e gravidade do envenenamento na região da picada por serpentes do gênero $B$ othrops ${ }^{14}$, e a recomendação quanto à dose de soro a ser administrada já não leva em conta a ocorrência de tal alteração $0^{5,7}$. I sso sugere que, embora a alteração da coagulação não seja um bom indicador de evol ução para mani festações locais, como a necrose, sua ausência pode indicar que o óbito é menos provável.

Entre os pacientes que faleceram por envenenamento crotálico, as percentagens de vômito, mialgia, mi ogl obinúria, sangramento e de alteração da coagulação sanguínea foram maiores do que as descritas para os pacientes vítimas desses acidentes. E studo prévio realizado com pacientes atendidos no HVB corrobora esses achados ${ }^{25}$. A utilização de maiores doses de soro no tratamento do paciente vítima de acidente crotálico do que naquele pi cado por Bothrops tem explicação nas orientações da 
Secretaria de Saúde de São Paulo, que tem recomendado doses maiores no envenenamento crotálico grave do que no envenenamento botrópico grave ${ }^{7,26}$.

\section{CONCLUSÃO}

A maioria dos acidentes por serpentes peçonhentas e de óbitos, no Estado de São Paulo, é causada por Bothrops, mas o envenenamento mais freqüentemente fatal é aquele provocado por Crotalus. Acidentes por Micrurus não são causas comuns de óbito nesse Estado. Picadas na perna associam-se com maior letalidade. O mesmo ocorre, no acidente por Bothrops, para picadas em pessoas mais idosas (50 anos e mais). Existe associação entre o tempo entre a picada e o atendimento médico com a letalidade do envenenamento. A complicação mais comum nos casos fatais é a insuficiência renal.

\section{AGRADE CIME NTOS}

Os autores agradecem aos profissionais de saúde que prestaram atendimento aos pacientes e aos responsáveis pelas unidades de saúde que, gentilmente, forneceram informações acerca dos casos.

\section{SUMMARY}

\section{Obits by venomous snakes in the State of São Paulo: evaluation of $\mathbf{4 3}$ cases from 1988 to 1993}

The prognostic factors and the causes for obit occurrence in ophidian envenoming are yet not completely clear.

PURPOSE. To determine the prognostic factors and the most probablecauses for obit occurrence in ophidian envenoming.

METHODS. In the State of São Paulo were notified 12,639 cases of accidents by venomous snakes from 1988 to 1993 . There were 43 deaths (0.34\%). The variables from the accident notification reports were compared with the promptuary notes and/ or with the death records (in lethal cases).

RESULTS. The snake genus was classified in 11,297 accidents and in 41 from the lethal ones. Bothrops was responsible for 9,828 (87\%) accidents and 28 (68.3\%) deaths, Crotalus for 1,359 (12.0\%) accidents and 13 deaths (31.7\%) and $\mathrm{Mi}$ crurus for 110 (1\%) accidents and no death $(p<0.05)$. The information regarding to sex and age of the patients was available, respectively, in 12,620 and 12,527 accidents and in all lethal ones. There were 9,783 (77.5\%) male patients in the accidents and $35(81.4 \%)$ in the lethal cases ( $p>$
0.05). Regarding to age, $15.9 \%$ of the patients and $41.8 \%$ of the ones who died were 50 years-old or more $(p<0.05)$. The most frequently bitten anatomic regions were: foot (42.2\%), hand (20.6\%), leg (17.6\%), and ankle (13.1\%) in the accidents, and foot (35.7\%) and leg (35.7\%) in the lethal cases $(p<0.05)$. Coagulation disorders occurred in 34 (91.9\%) from 37 patients who died (those where this datum was available). The information above was not available in non lethal cases. The most common manifestations and complications implicated as possible death causes were: acute renal failure (34- 79.1\%), acute respiratory failure (28$65.1 \%)$, shock (18-41.9\%), and sepsis (18-41.9\%). Among all lethal cases but one without information, $29.4 \%$ of the patients died within the first two days after bite and $67.6 \%$ within the first 5 days. Acute respiratory failure was most common among the patients who died owing to crotalic envenoming, and sepsis was only seen in bothropic envenoming.

ConCLUSION. Most of the accidents and obits are caused by Bothrops; Crotalus envenoming, leg bites, and accidents in 50 year-old patients (or more) are frequently lethal; the most common complication in lethal cases is acute renal failure. [Rev Ass Med Brasil 1998; 44(4): 312-8.]

KEY WORDS: Snake bite. Clinical features. Obit.

\section{REFERÊNCIAS BIBLIOGRÁFICAS}

1. Hoge AR, Romano-Hoge SARWL. Sinopse das serpentes peçonhentas doBrasil. MemInst Butantan 1978/79; 42/43: 373-496.

2. Ribeiro LA, Pires de Campos VAF, Albuquerque MJ , Takaoka NY. Acidente ofídico no Estado de São Paulo. Rev Ass Med Brasil 1993; 39: 4-7.

3. J orge MT, Ribeiro LA. Acidentes por serpentes peçonhentas do Brasil. Rev Ass Med Brasil 1990; 36: 66-77.

4. J orge MT, Ribeiro LA, Silva MLR, KusanoEJ U, Mendonça J S Microbiological studies of abscesses complicating Bothrops snakebite in humans: a prospective study. Toxicon 1994; 32: 743-8.

5. Brasil. Ministério da Saúde. Manual de diagnóstico e tratamento de acidentes ofídicos. Brasília, Centro de Documentação do Ministério da Saúde, 1987.

6. J orge MT, Ribeiro LA. Epidemiologia e quadro clínico do acidente por cascavel sul-americana (Crotalus durissus). Rev Inst Med Trop São Paulo 1992; 34: 347-54.

7. São Paulo, Secretaria de Saúde do Estado de São Paulo. Manual de vigilância epidemiológica; acidentes por animais peçonhentos; identificação, diagnóstico e tratamento. Centro de Vigilância E pidemiológica "Professor Alexandre Vranjac", Instituto Butantan. Secretaria de Estado da Saúde, 1993.

8. Rosenfeld G. Moléstias por venenos animais. Pinheiros Ter 1965; 17: 3-15.

9. Rosenfeld G. Acidentes por animais peçonhentos: serpentes, aranhas, escorpiões. In Veronesi R. (ed). Doenças infecciosas eparasitárias, 8a ed. RiodeJ aneiro, Guanabara Koogan, 1991; 951-62. 
10. Barroso S. Mordeduras de cobra e seu tratamento. Rio de J aneiro, Laemmert, 1944.

11. Ribeiro LA, J orge MT. Epidemiologia e quadro clínico dos acidentes por serpentes Bothrops jararaca adultas e filhotes. Rev Inst Med Trop São Paulo 1990; 32: 436-42.

12. Ribeiro LA, J orge MT. Acidente por serpentes do gênero Micrurus ("coral"). Análise de 7 casos. Rev Soc Bras Med Trop 1986; 19(supl.): 28.

13. Fonseca F . Animais peçonhentos. São Paulo, E mpresa Gráfica da "Revista dos Tribunais", 1949.

14. Ribeiro LA, J orge MT. Fatores prognósticos da evolução das manifestações locais em acidentes por serpentes do gênero Bothrops. Rev Soc Bras Med Trop 1989; 22 (supl.): 68-9. (Resumo 177)

15. J orgeMT, RibeiroLA. Acidentes por animais peçonhentos. In. Amato NetoV, BaldyJ LS (eds). Doençastransmissíveis. 3a ed. São Paulo, Sarvier, 1989; .133-41.

16. Ribeiro LA, J orge MT. Acidentes por serpentes do gênero Bothrops: série de 3.139 casos. Rev Soc Bras Med Trop 1997, 30(6): 475-80.

17. Lebrão $M L$, Ribeiro LA, J orge $M T$. Avaliação dos óbitos por serpentes peçonhentas no Estado de São Paulo, 1988/1989. Rev Ass Med Brasil 1995; 41: 342-7.

18. Amaral CFS, Resende NA, Silva OA et al. Insuficiência renal aguda secundária a acidentes ofídicos botrópico e crotálico. Análise de 63 casos. Rev Inst Med Trop São Paulo 1986; 28: 220-7.
19. Azevedo Marques MM, Hering SE, Cupo P. Evidence that Crotalus durissus terrificus (South american rattlesnake) envenomation in humans causes myolysis rather than hemoIysis. Toxicon 1987; 25: 1.163-8.

20. Craven DE, Steger KA, Ducan RA. Prevention and control of nosocomial pneumonia. In Wenzel RP (ed). Prevention and control of nosocomial infections. Baltimore, Williams \& Wilkins, 1993; 580-99.

21. Lopez M, Foscarini LG, Alvares J M et al. Tratamento intensivo das complicações do acidente ofídico. Rev Assoc Méd Minas Gerais 1972; 23: 107-12.

22. Rosenfeld G. Symptomatology, pathology, and treatment of snake bites in South America, In Bucherl W, Buckley EE (eds). Venomous animals and their venoms. New York, Academic Press, 1971; 345-84.

23. Kouyoumdjian J A, Polizeli C. Acidente ofídico causado por Bothrops moojeni: relato de 37 casos. Rev Inst Med Trop São Paulo 1988; 30: 424-32.

24. Ribeiro LA, J orge MT. Fatores prognósticos para óbito em envenenamento botrópico. Rev Bras Tox 1995; 8: 277. (Resumo, 4.6.98)

25. J orge MT, Ribeiro LA. Fatores prognósticos no acidente por Crotalus durissus terrificus. Rev Soc Bras Med Trop 1989; 22(supl.): 69 (Resumo, 178)

26. São Paulo. Secretaria de Estado da Saúde de São Paulo. Instituto Butantan. Manual para o diagnóstico e tratamento de acidentes por animais peçonhentos, 1982. 